\title{
Circulating Interleukin-37 Levels in Rheumatoid Arthritis and Systemic Lupus Erythematosus and Their Correlations With Disease Activity: A Meta-analysis
}

\author{
Young Ho Lee, M.D., Ph.D., Gwan Gyu Song, M.D., Ph.D. \\ Division of Rheumatology, Department of Internal Medicine, Korea University College of Medicine, Seoul, Korea
}

Objective. To assess the circulating levels of interleukin (IL)-37 in rheumatoid arthritis (RA) and systemic lupus erythematosus (SLE), and determine a correlation between plasma/serum IL-37 levels and disease activity. Methods. We performed a meta-analysis comparing plasma/serum IL-37 level between patients with RA or SLE and controls, and examined correlation coefficients between circulating IL-37 levels and disease activity. Results. A total of 14 publications included 711 patients with RA and 394 controls, 522 patients with SLE and 259 controls. In the RA group, the IL-37 level was significantly higher than in the control group (standardized mean difference $[\mathrm{SMD}]=1.222,95 \%$ confidence interval $[\mathrm{Cl}]=0.722 \sim 1.711, \mathrm{p}<0.001$ ). Subgroup analysis by sample size showed a significantly higher IL-37 level in RA group of large $(\mathrm{n}>90)$ and small sample numbers $(\mathrm{n} \leq 90)(\mathrm{SMD}=0.994,95 \% \mathrm{Cl}=0.323 \sim 1.666, \mathrm{p}<0.001 ; \mathrm{SMD}=1.617,95 \% \mathrm{Cl}=1.328 \sim 1.906, \mathrm{p}<0.001)$. In addition, IL-37 level in SLE group was significantly higher than in control group ( $\mathrm{SMD}=1.096,95 \% \mathrm{Cl}=0.635 \sim 1.558, \mathrm{p}<0.001$ ). A strong association between circulating IL-37-level and RA activity based on Disease activity Score 28 was shown (correlation coefficients $=0.547,95 \% \mathrm{Cl}=0.355 \sim 0.695, \mathrm{p}<0.001)$. Meta-analysis of the coefficients for correlation indicated a positive correlation between the circulating level of IL-37 and SLE activity based on Systemic Lupus Erythematosus Disease Activity Index (coefficients for correlation $<0.588,95 \% \mathrm{Cl}=0.270 \sim 0.806, \mathrm{p}=0.003$ ). Conclusion. Our meta-analysis showed that circulating IL-37 levels are higher in RA and SLE patients, and there is a positive correlation between IL-37 and disease activity in RA and SLE. (J Rheum Dis 2020;27:152-158)

Key Words. IL37 protein, human, Rheumatoid arthritis, Systemic lupus erythematosus

\section{INTRODUCTION}

Rheumatoid arthritis (RA) is a chronic inflammatory autoimmune disease, which mainly affects the synovial joints and causes synovial cell hyperplasia and the development of new blood vessels; leading to bone erosion and joint deformation [1]. Systemic lupus erythematosus (SLE) is a prototypical autoimmune disease defined by B cell hyperactivity, high production of autoantibody, immune-complex deposition, and several organ damage [2]. While the cause of these diseases is not fully understandable, the imbalance between anti-inflammatory and pro-inflammatory cytokines by elevating pro-inflammatory cytokines and/or reducing anti-inflammatory cytokines lead to autoimmune pathogenesis like RA and SLE [3].

Interleukin-37 (IL-37), a member of the Interleukin-1 (IL-1) family, was first identified as a new molecule in the IL-1 family locus on chromosome 2q13 [4]. Initially it was known as IL-1 family member 7 (IL-1F7), but later its name was changed to IL-37 [5]. IL-37 is an anti-inflammatory cytokine that suppress immune responses by reducing the production of pro-inflammatory cytokines like tumor necrosis factor- $\alpha$, IL-1 $\alpha$, IL-1 $\beta$, and IL-6 [6].

\footnotetext{
Received : January 30, 2020, Revised : February 21, 2020, Accepted : March 10, 2020

Corresponding to : Young Ho Lee (iDhttp://orcid.org/0000-0003-4213-1909

Division of Rheumatology, Department of Internal Medicine, Korea University Anam Hospital, Korea University College of Medicine, 73 Goryeodae-ro, Seongbuk-gu, Seoul 02841, Korea. E-mail : lyhcgh@korea.ac.kr
}

Copyright (c) 2020 by The Korean College of Rheumatology.

This is an Open Access article, which permits unrestricted non-commerical use, distribution, and reproduction in any medium, provided the original work is properly cited. 
IL-37 can significantly reduce the production of inflammatory cytokines in LPS-stimulated mouse primary macrophages [7]. The inhibitory impact of IL-37 in autoimmune disease pathogenesis was also confirmed in mice models of RA and SLE [8]. Nonetheless, IL-37's role in pathogenesis of RA and SLE in human remains uncertain. Studies have shown high circulating IL-37 levels in patients with RA or SLE and the correlation between IL-37 levels and disease activity, but the results from individual studies varied in terms of the magnitude of the effect sizes [9-22]. This may be due to a small sample size, weak statistical power and/or clinical heterogeneity. We conducted this meta-analysis to overcome the limits in individual studies and to resolve the inconsistencies. IL-37 plays an important role IL-37 in a number of inflammatory and autoimmune diseases and both RA and SLE share inflammatory and autoimmune profiles and [6]. The aim of this research was to determine the circulating IL-37 levels in patients with RA or SLE relative to those in healthy controls and to assess their association with disease activity.

\section{MATERIALS AND METHODS}

\section{Identification of eligible studies and data extraction}

We conducted a literature search for studies that assessed the status of IL-37 in RA or SLE and controls and/or reports that measured the relationship between circulating levels of IL-37 (serum or plasma) and disease activity. To identify all previous articles available (up to November 2019), PUBMED, EMBASE and Cochrane databases were checked for. In the search, the following key words and subject terms were used: "IL-37," "level OR activity OR correlation," "rheumatoid arthritis," and "systemic lupus erythematosus." Additional studies not identified by the above-mentioned online databases were also checked for all references cited. Studies were considered eligible if: (1) case-control studies or cross-sectional trials were conducted; (2) data were given on IL-37 levels in case and control groups; and/or (3) data were provided on the relation between circulating IL-37 levels and disease activity based on Disease Activity Score 28 (DAS28) or Systemic Lupus Erythematosus Disease Activity Index (SLEDAI). Studies were excluded if: (1) contained data that were overlapping or insufficient, or (2) review article or reported cases. Two independent researchers collected data on methodology and findings from the original studies. Any discrepancies between reviewers were solved by con- sensus, and the meta-analysis was carried out in accordance with the guidelines of PRISMA [23]. From each analysis, the following information was extracted: primary author, year of publication, country ethnicity, number of participants, mean and standard deviation (SD) of level IL-37, and coefficients of correlation between level IL-37 and level RA or SLE. When the data were medians, interquartile ranges or ranges, the mean and SD were determined using the formulae previously described $[24,25]$.

\section{Evaluation of statistical associations}

We conducted a meta-analysis that examined the relationship between levels of IL-37 and RA or SLE. Results were presented as standardized mean differences (SMDs) or as coefficients of correlation and confidence intervals (CIs) of 95\% for data continuity. By dividing the mean difference between two groups by the pooled SD, SMDs were measured and used when multiple scales were combined to quantify the same definition. This measurement compares case and arms based on standardized values. This was the magnitude of SMD: 0.2 0.5, small effect; $0.5 \sim 0.8$, medium effect; $\geq 0.8$, large effect [26]. We also used Cochran's Q-statistics to test differences and heterogeneities within and between the studies [27]. The heterogeneity method was used to determine the null hypothesis that the same effect was tested by all experiments. The random effects model was chosen for meta-analysis, when the relevant $\mathrm{Q}$-statistic $(\mathrm{p}<0.10)$ revealed heterogeneity across studies [28]. Otherwise, the fixed effects model was used, assuming that all studies had the same underlying effect estimated and considered only variation within the study [27]. We quantified the impact of heterogeneity using $I^{2}=100$ percent $(\mathrm{Q}-\mathrm{df}) / \mathrm{Q}[29]$, where $I^{2}$ calculated the incoherence between studies and whether the percentage of total differences between studies was attributable to heterogeneity instead of chance. $I^{2}$ ranged from $0 \%$ to $100 \%$; $I^{2}$ ranged from $25 \%, 50 \%$, and $75 \%$, respectively, from weak, moderate, and strong estimates [29]. The Comprehensive Meta-Analysis Computer Program (Biostat Inc., Englewood, NJ, USA) was used to perform statistical manipulations.

\section{Evaluation of heterogeneity, sensitivity test, and publication bias}

A subgroup analysis of sample size was carried out in order to examine possible sources of heterogeneity detected in the meta-analysis. A sensitivity test was performed in order to evaluate the influence of each study on the 
pooled odds ratio (OR) by ignoring each study. We explored publication biases by using funnel plots and the linear regression test of Egger to calculate funnel plot asymmetry [30].

\section{RESULTS}

\section{Studies included in the meta-analysis}

We have identified 271 studies using manual and electronic search methods. Seventeen of these studies have been chosen based on the title and abstract for full-text analysis. Of those 17 studies, 3 were excluded, because no
IL-37 data or duplicate data existed. Thus, 14 articles fulfilled the criteria for inclusion [9-22]. One of the eligible studies contained data from two separate groups [9]. These articles included 711 RA and 394 controls and 522 SLE and 259 controls (Table 1). Eight of these studies examined RA and control groups for circulatory IL-37 levels, and four studies provided correlation coefficients between IL-37 and RA activities. Six of these studies investigated SLE and control groups in circulating IL-37 levels; four studies provided correlation coefficients between IL-37 and the SLE activities. Table 1 summarizes selected characteristics of the association between IL-37 and stud-

Table 1. Characteristics of individual studies included in the meta-analysis

\begin{tabular}{|c|c|c|c|c|c|c|c|c|c|}
\hline \multirow[t]{2}{*}{ Authors } & \multirow[t]{2}{*}{ Country } & \multirow[t]{2}{*}{ Ethnicity } & \multirow[t]{2}{*}{ Disease } & \multicolumn{2}{|c|}{ Number } & \multicolumn{2}{|c|}{$\begin{array}{c}\text { IL-37 level } \\
\text { (pg/mL or ng/L) }\end{array}$} & \multirow{2}{*}{$\begin{array}{l}\text { Correlation } \\
\text { coefficient* }\end{array}$} & \multirow{2}{*}{$\begin{array}{c}\text { Disease } \\
\text { activity score }\end{array}$} \\
\hline & & & & Case & Control & Case & Control & & \\
\hline Yuan et al., 2019 [9] & China & Asian & RA & 65 & 60 & 712.40 & 133.50 & 0.459 & DAS28 \\
\hline Ragab et al., 2019 [10] & Egypt & Arab & RA & 48 & 42 & 398.90 & 101.90 & 0.746 & DAS28 \\
\hline Rodriguez et al., 2018 [22] & Spain & European & RA & 104 & 33 & 501.15 & 358.17 & $(-)$ & $(-)$ \\
\hline Song et al., 2018 [11] & China & Asian & RA & 59 & 46 & 139.50 & 114.20 & $(-)$ & $(-)$ \\
\hline Yang et al., 2015 [12] & China & Asian & RA & 152 & 100 & 284.70 & 84.64 & $(-)$ & $(-)$ \\
\hline Xia et al., 2015 [21] & China & Asian & RA & 150 & 50 & 548.90 & 214.80 & $(-)$ & $(-)$ \\
\hline Ye et al., 2015 [13] & China & Asian & RA & 49 & 33 & 205.00 & 105.50 & $(-)$ & $(-)$ \\
\hline Xia et al.-1, 2015 [14] & China & Asian & RA & 34 & $(-)$ & $(-)$ & $(-)$ & 0.348 & DAS28 \\
\hline Zhao et al., 2014 [15] & China & Asian & RA & 50 & 30 & 649.50 & 55.25 & 0.545 & DAS28 \\
\hline Yuan et al., 2019 [9] & China & Asian & SLE & 107 & 60 & 300.65 & 133.50 & $(-)$ & $(-)$ \\
\hline Tawfik et al., 2017 [16] & Egypt & Arab & SLE & 100 & 50 & 288.64 & 44.53 & 0.600 & SLEDAI \\
\hline Godsell et al., 2016 [17] & Australia & European & SLE & 129 & $(-)$ & $(-)$ & $(-)$ & 0.218 & SLEDAI \\
\hline Wu et al., 2016 [18] & China & Asian & SLE & 90 & 78 & 13.20 & 10.11 & $(-)$ & $(-)$ \\
\hline Ye et al., 2014 [19] & China & Asian & SLE & 66 & 41 & 202.75 & 103.52 & 0.342 & SLEDAI \\
\hline Song et al., 2013 [20] & China & Asian & SLE & 30 & 30 & 24.30 & 15.15 & 0.910 & SLEDAI \\
\hline
\end{tabular}

IL: interleukin, RA: rheumatoid arthritis, SLE: systemic lupus erythematosus, (-): not available, DAS28: Disease Activity Score 28, SLEDAI: Systemic Lupus Erythematosus Disease Activity Index. * Correlation coefficient between IL-37 and disease activity in RA or SLE.

Table 2. Meta-analysis of the association between circulating IL-37 levels and RA or SLE

\begin{tabular}{|c|c|c|c|c|c|c|c|c|c|}
\hline \multirow{2}{*}{ Disease } & \multirow{2}{*}{ Groups } & \multirow{2}{*}{ Population } & \multirow{2}{*}{$\begin{array}{l}\text { No. of } \\
\text { studies }\end{array}$} & \multicolumn{3}{|c|}{ Test of association } & \multicolumn{3}{|c|}{ Test of heterogeneity } \\
\hline & & & & SMD* & $95 \% \mathrm{Cl}$ & $p$-value & Model & p-value & $I^{2}$ \\
\hline \multirow[t]{3}{*}{ RA } & All & Overall & 8 & 1.222 & $0.722 \sim 1.711$ & $<0.001$ & $\mathrm{R}$ & $<0.001$ & 91.8 \\
\hline & Sample size & Large $(\mathrm{n}>90)$ & 5 & 0.994 & $0.323 \sim 1.666$ & 0.004 & $\mathrm{R}$ & $<0.001$ & 94.2 \\
\hline & & Small $(n \leq 90)$ & 3 & 1.617 & $1.328 \sim 1.906$ & $<0.001$ & $\mathrm{~F}$ & 0.394 & 0 \\
\hline \multirow[t]{3}{*}{ SLE } & All & Overall & 5 & 1.096 & $0.635 \sim 1.558$ & $<0.001$ & $\mathrm{R}$ & $<0.001$ & 85.9 \\
\hline & Sample size & Large $(\mathrm{n}>90)$ & 4 & 1.150 & $0.598 \sim 1.702$ & $<0.001$ & $\mathrm{R}$ & $<0.001$ & 89.2 \\
\hline & & Small $(\mathrm{n} \leq 90)$ & 1 & 0.858 & $0.329 \sim 1.386$ & 0.001 & NA & $\mathrm{NA}$ & NA \\
\hline
\end{tabular}

IL: interleukin, RA: rheumatoid arthritis, SLE: systemic lupus erythematosus, SMD: standard mean difference, Cl: confidence interval, R: random effect model, F: fixed effects model, NA: not available. *Magnitude of Cohen's d effect size (SMD), where 0.2 to 0.5 is a small effect, 0.5 to 0.8 is a medium effect, and $\geq 0.8$ is a large effect. 
ies of RA and SLE.

\section{Meta-analysis comparing the circulating IL-37 level in RA, SLE, and controls}

The RA group was significantly higher than the control group $(\mathrm{SMD}=1.222,95 \% \mathrm{CI}=0.722 \sim 1.711, \mathrm{p}<0.001$ ) as compared to the control group (Table 2, Figure 1). The sample size subgroup test showed that the IL-37 levels in the large $(n>90)$, and small size $(n \leq 90)$. RA group were significantly higher than in the control group $(\mathrm{SMD}=$ 0.994, 95\% CI $=0.323 \sim 1.666, \mathrm{p}<0.001$; $\mathrm{SMD}=1.617$, $95 \% \mathrm{CI}=1.328 \sim 1.906, \mathrm{p}<0,001$ ) (Table 2). The level of IL-37 was also significantly higher within SLE than within the control group $(\mathrm{SMD}=1.096,95 \% \mathrm{CI}=0.635 \sim$
1.558, $\mathrm{p}<0.001)$. The subgroup sample size analysis showed that in the SLE groups of large and small sample numbers the IL-37 level were significantly higher than that in the controls $(\mathrm{SMD}=1.150,95 \% \mathrm{CI}=0.598 \sim$ 1.702, $\mathrm{p}<0.001 ; \mathrm{SMD}=0.858,95 \% \mathrm{CI}=0.329 \sim 1.386$, $\mathrm{p}=0.001)$ (Table 2).

\section{Meta-analysis of the relationship between circulating IL-37 level and activity of RA and SLE}

A substantially positive correlation between the IL-37 and DAS28-based RA activity was shown by meta-analysis of correlation coefficients (correlation coefficients = $0.547,95 \% \mathrm{CI}=0.355 \sim 0.695, \mathrm{p}<0.001$ ) (Table 3, Figure 2 ). In addition, a substantially positive association of

A

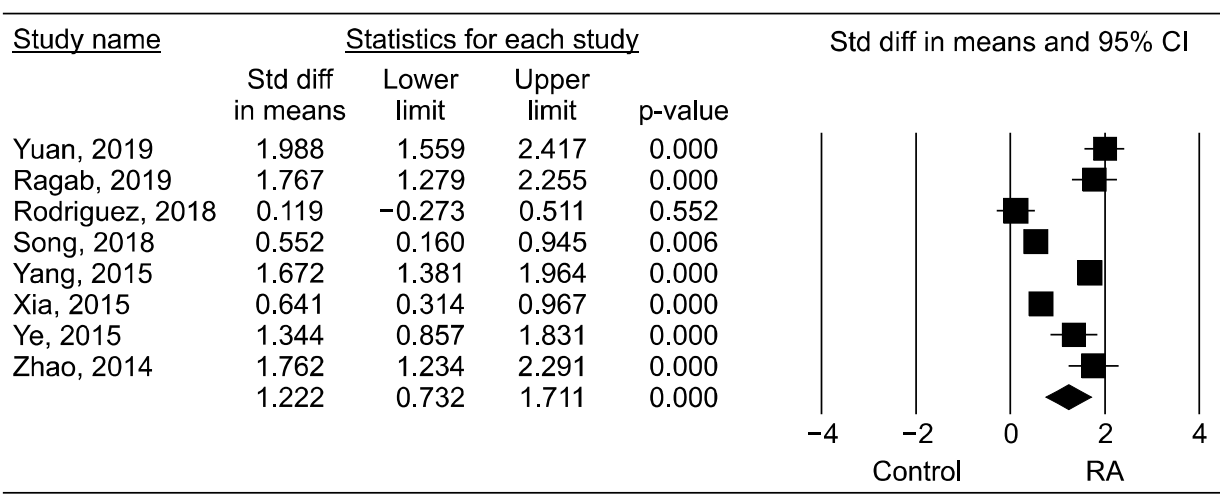

\section{B}

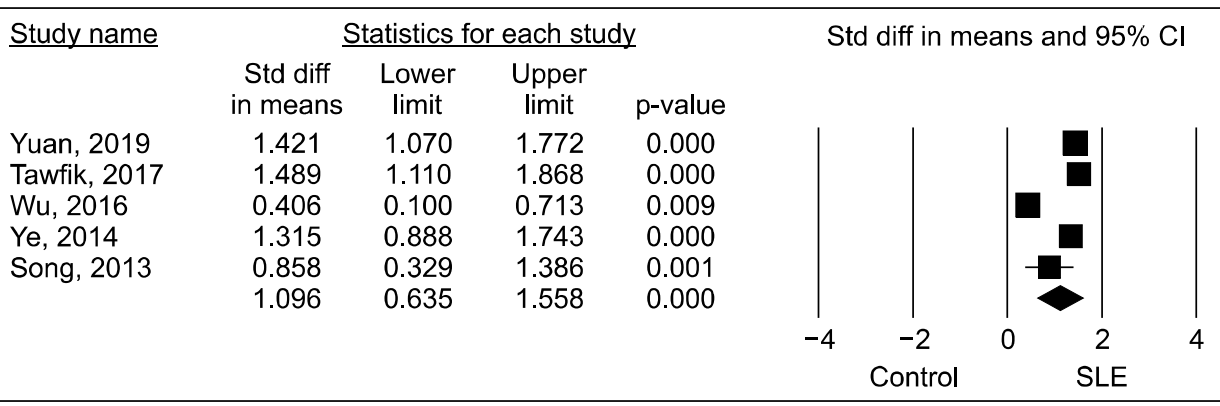

Figure 1. Meta-analysis of the relationship between interleukin37 levels and rheumatoid arthritis (RA) (A) or systemic lupus erythematosus (SLE) (B). Std diff: standard difference, $\mathrm{CI}$ : confidence interval.

Table 3. Meta-analysis of the correlation coefficient between circulating IL-37 levels and RA or SLE activity

\begin{tabular}{|c|c|c|c|c|c|c|c|c|}
\hline \multirow[b]{2}{*}{ Disease } & \multirow{2}{*}{$\begin{array}{l}\text { Disease } \\
\text { activity }\end{array}$} & \multirow{2}{*}{$\begin{array}{l}\text { No. of } \\
\text { studies }\end{array}$} & \multicolumn{3}{|c|}{ Test of association } & \multicolumn{3}{|c|}{ Test of heterogeneity } \\
\hline & & & $\begin{array}{l}\text { Correlation } \\
\text { coefficient* }\end{array}$ & $95 \% \mathrm{Cl}$ & p-value & Model & p-value & $I^{2}$ \\
\hline RA & DAS28 & 4 & 0.547 & $0.355 \sim 0.695$ & $<0.001$ & $\mathrm{R}$ & 0.040 & 63.9 \\
\hline SLE & SLEDAI & 4 & 0.588 & $0.270 \sim 0.806$ & 0.003 & $\mathrm{R}$ & $<0.001$ & 93.0 \\
\hline
\end{tabular}

IL: interleukin, RA: rheumatoid arthritis, SLE: systemic lupus erythematosus, Cl: confidence interval, DAS28: Disease Activity Score 28, SLEDAI: Systemic Lupus Erythematosus Disease Activity Index, R: random effects model, *Correlation coefficient between IL-37 and disease activity in RA or SLE. 
A

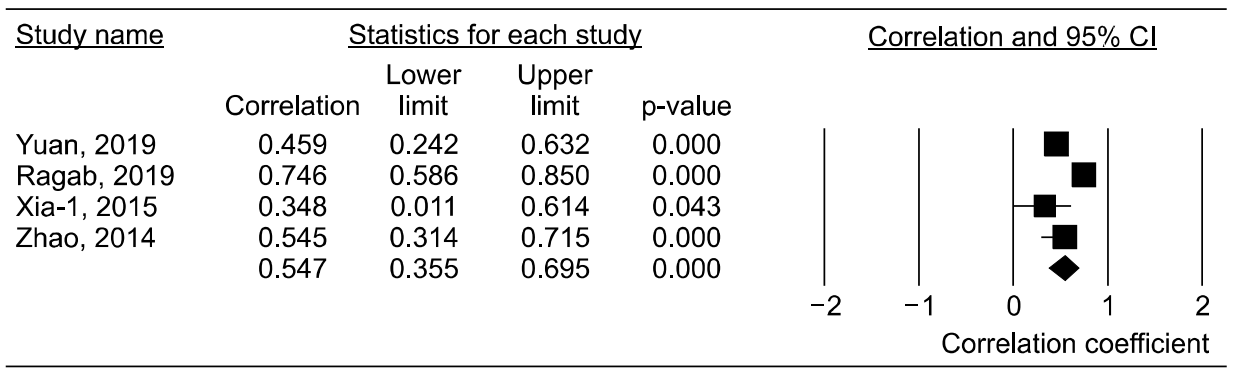

B

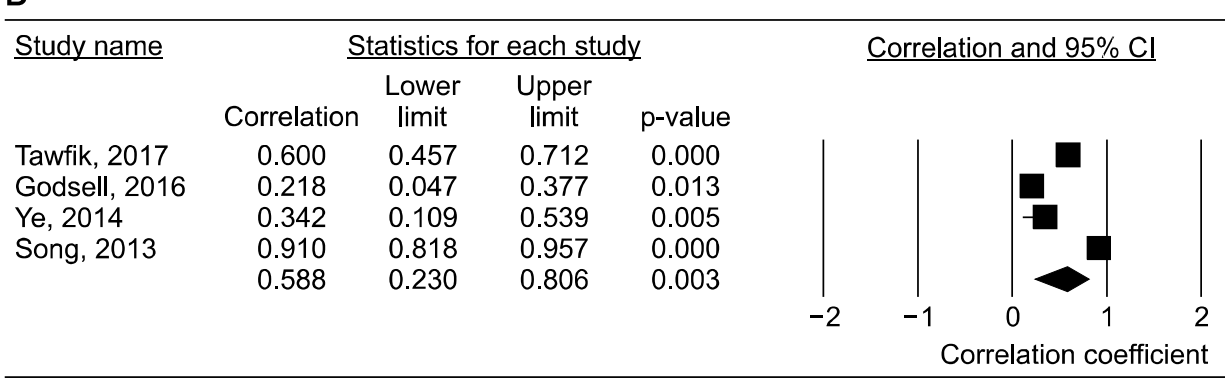

Figure 2. Meta-analysis of correlation coefficients between interleukin-37 levels and the activity of rheumatoid arthritis (A) or systemic lupus erythematosus (B). CI: confidence interval.

IL-37 and SLE activity on the basis of SLEDAI has been demonstrated (correlation coefficients $=0.588$, 95\% $\mathrm{CI}=0.270 \sim 0.806$ and $\mathrm{p}=0.003$ ) in meta-analysis (Table 3, Figure 2).

\section{Heterogeneity, sensitivity test, and publication bias}

During IL-37 status meta-analysis in RA and SLE, inter-study heterogeneity was reported (Tables 2 and 3). Although high heterogeneity remained in the large sample group following the sample size analysis of the subgroup, the directions of SMDs were the same and the main cause of heterogeneity was due to the difference in SMD magnitude. The analysis of the subgroup did not show any heterogeneity in the small sample size group (Table 2). Sensitivity analysis has shown that no single study affected the pooled SMD significantly, indicating that the results of this meta-analysis were robust. Publication biases can lead to disproportionate numbers of positive studies and present a meta-analysis problem. No evidence of publication bias (Egger regression test p-values $>0.1$ ) was recorded by the Egger regression test.

\section{DISCUSSION}

Autoimmune disorders are caused by immune responses, which generate aberrant antibodies and attack the cells and tissues of the body [31]. IL-37 is an anti-inflammatory cytokine expressed in a number of immune and non-immune cells and IL-37 decreases both innate and acquired inflammation [4]. In regulating inflammatory response, IL-37 is the key cytokine by inhibiting expression, production and function of proinflammatory cytokines [6]. Transgenic mice IL-37 can be secured against autoimmune disease development [8]. In a range of inflammatory and autoimmune diseases, IL-37 is thought to play its potential role by suppressing immune responses and inflammation [5]. However, its role is not well understood in RA and SLE. We have combined evidence for circulating IL-37 levels in RA and SLE, and the correlation between plasma/serum IL-37 and disease activity in this meta-analysis. The meta-analysis of 14 studies showed that the circulating IL-37 levels in the RA or SLE group were significantly higher than in the control group. IL-37 had a positive correlation to RA or SLE, which showed an increased level of IL-37 activity. The meta-analysis evidence suggested that circulating IL-37 could influence the pathogenesis of RA and SLE unequivocally. It also supported the idea that IL-37 is involved in pathogenesis of RA and SLE.

Our results showed serum/plasma IL-37 levels increased in RA or SLE patients and were strongly and positively linked to disease activity. It is therefore reasonable to speculate that proinflammatory cytokines in patients with RA or SLE can encourage IL-37 expression, since IL-37 mediates a negative feedback mechanism in these patients to suppress excessive pro-inflammatory cytokines. The rise in anti-inflammatory cytokines such as IL-37 can 
be a fundamental mechanism for alleviating inflammation. Our results suggested that the inflammatory process in RA and SLE may trigger this cytokine, and high levels of IL-37 can decline the active inflammatory process. By eliminating the production of inflammatory cytokines, IL-37 may be an important cytokine in the control of both RA and SLE pathogeneses. These observations implied that IL-37 probably played an important role in inhibiting pathogenesis of RA and SLE. IL-37 can be a biomarker in RA or SLE through this meta-analysis, although the underline mechanism has not been understood. Future efforts are necessary in order to identify IL-37 regulatory mechanisms for mediating RA and SLE immune reactions.

IL-37 has anti-arthritic activity in RA patients and collagen-induced arthritis mice [13], and low doses of IL-37 decreased joint inflammation, and significantly reduced synovial IL- $1 \beta$, TNF- $\alpha$, IL-6, CCL3, CXCL1 or MIP- $1 \alpha$ in mice [32]. Such reductions have been associated with recruiting less neutrophils into the joint, suggesting that IL-37 is a crucial anti-inflammatory cytokine in regulating pathogenesis of RA. In addition, plasma IL-37 levels in SLE patients were upregulated and associated with anti-Sm, anti-RNP and C3 [18], and the rise in IL-37 levels in SLE patients was correlated with inflammatory cytokines, elevated disease activity, and involvement in mucocutaneous and renal conditions [16]. Furthermore, IL-37 has an important part to play in regulating SLE pathogenesis by inhibiting inflammatory cytokine production [19]. Thus, IL-37 could provide a new target for the study of pathogenesis and SLE treatment.

This meta-analysis has some drawbacks that must be taken into consideration. First, the majority of studies had small samples, and only a small number of studies have assessed the correlation of the IL-37 levels with the activity of the disease. The meta-analysis can therefore be underpowered. Second, the studies included in this meta-analysis were heterogeneous in population and clinical characteristics. Therefore, our results may have affected the heterogeneity, confounding factors and limited clinical information provided by the study population. Such limited data did not allow further research, although we performed a sensitivity test and evaluated several subgroups. This meta-analysis, however, also has its strength. Our meta-analysis is the first to provide composite evidence for IL-37 status in patients with RA or SLE, to the best of our knowledge. Our data on the relationship between IL-37 levels and RA or SLE achieved greater precision compared to individual studies, due to the in- creased statistical power and resolution of independent analytic results.

\section{CONCLUSION}

In conclusion, our meta-analysis has shown that IL-37 circulatory levels are higher in RA and SLE patients and that there is a considerably positive correlation between IL-37 and disease activity. Therefore, our meta-analysis shows that IL-37 is likely to play a role in RA and SLE, although further studies are needed to understand the mechanism by which IL-37 levels directly contribute to RA and SLE pathogenesis.

\section{CONFLICT OF INTEREST}

No potential conflict of interest relevant to this article was reported.

\section{AUTHOR CONTRIBUTIONS}

Y.H.L. was involved in conception and design of study, acquisition of data, analysis and/or interpretation of data, drafting the manuscript, revising the manuscript critically for important intellectual content. G.G.S. was involved in conception and design of study, analysis and/or interpretation of data, drafting the manuscript.

\section{REFERENCES}

1. Mellado M, Martínez-Muñoz L, Cascio G, Lucas P, Pablos JL, Rodríguez-Frade JM. T cell migration in rheumatoid arthritis. Front Immunol 2015;6:384.

2. Ruiz-Irastorza G, Khamashta MA, Castellino G, Hughes GR. Systemic lupus erythematosus. Lancet 2001;357: 1027-32.

3. Mateen S, Zafar A, Moin S, Khan AQ, Zubair S. Understanding the role of cytokines in the pathogenesis of rheumatoid arthritis. Clin Chim Acta 2016;455:161-71.

4. Boraschi D, Lucchesi D, Hainzl S, Leitner M, Maier E, Mangelberger D, et al. IL-37: a new anti-inflammatory cytokine of the IL-1 family. Eur Cytokine Netw 2011;22:127-47.

5. Dinarello CA. Overview of the IL-1 family in innate inflammation and acquired immunity. Immunol Rev 2018; 281:8-27.

6. Jia H, Liu J, Han B. Reviews of interleukin-37: functions, receptors, and roles in diseases. Biomed Res Int 2018;2018: 3058640.

7. Zhao M, Li Y, Guo C, Wang L, Chu H, Zhu F, et al. IL-37 isoform $\mathrm{D}$ downregulates pro-inflammatory cytokines expression in a Smad3-dependent manner. Cell Death Dis 2018;9:582.

8. Ye L, Huang Z. IL-37 restrains autoimmune diseases. 
Oncotarget 2015;6:21775-6.

9. Yuan ZC, Wang JM, Huang AF, Su LC, Li SJ, Xu WD. Elevated expression of interleukin-37 in patients with rheumatoid arthritis. Int J Rheum Dis 2019;22:1123-9.

10. Ragab D, Mobasher S, Shabaan E. Elevated levels of IL-37 correlate with T cell activation status in rheumatoid arthritis patients. Cytokine 2019;113:305-10.

11. Song L, Wang Y, Sui Y, Sun J, Li D, Li G, et al. High interleukin-37 (IL-37) expression and increased mucin-domain containing-3 (TIM-3) on peripheral T cells in patients with rheumatoid arthritis. Med Sci Monit 2018;24:5660-7.

12. Yang L, Zhang J, Tao J, Lu T. Elevated serum levels of Interleukin-37 are associated with inflammatory cytokines and disease activity in rheumatoid arthritis. APMIS 2015; 123:1025-31.

13. Ye L, Jiang B, Deng J, Du J, Xiong W, Guan Y, et al. IL-37 alleviates rheumatoid arthritis by suppressing IL-17 and IL-17-triggering cytokine production and limiting Th17 cell proliferation. J Immunol 2015;194:5110-9.

14. Xia T, Zheng XF, Qian BH, Fang H, Wang JJ, Zhang LL, et al. Plasma interleukin-37 is elevated in patients with rheumatoid arthritis: its correlation with disease activity and Th1/Th2/Th17-related cytokines. Dis Markers 2015;2015: 795043.

15. Zhao PW, Jiang WG, Wang L, Jiang ZY, Shan YX, Jiang YF. Plasma levels of IL-37 and correlation with TNF- $\alpha$, IL-17A, and disease activity during DMARD treatment of rheumatoid arthritis. PLoS One 2014;9:e95346.

16. Tawfik MG, Nasef SI, Omar HH, Ghaly MS. Serum interleukin-37: a new player in lupus nephritis? Int J Rheum Dis 2017;20:996-1001.

17. Godsell J, Rudloff I, Kandane-Rathnayake R, Hoi A, Nold MF, Morand EF, et al. Clinical associations of IL-10 and IL-37 in systemic lupus erythematosus. Sci Rep 2016;6: 34604.

18. Wu GC, Li HM, Wang JB, Leng RX, Wang DG, Ye DQ. Elevated plasma interleukin-37 levels in systemic lupus erythematosus patients. Lupus 2016;25:1377-80.

19. Ye L, Ji L, Wen Z, Zhou Y, Hu D, Li Y, et al. IL-37 inhibits the production of inflammatory cytokines in peripheral blood mononuclear cells of patients with systemic lupus erythematosus: its correlation with disease activity. J Transl Med
2014;12:69.

20. Song L, Qiu F, Fan Y, Ding F, Liu H, Shu Q, et al. Glucocorticoid regulates interleukin-37 in systemic lupus erythematosus. J Clin Immunol 2013;33:111-7.

21. Xia L, Shen H, Lu J. Elevated serum and synovial fluid levels of interleukin-37 in patients with rheumatoid arthritis: attenuated the production of inflammatory cytokines. Cytokine 2015;76:553-7.

22. Rodríguez-Carrio J, Alperi-López M, López P, Ballina-García FJ, Suárez A. Profiling of B-cell factors and their decoy receptors in rheumatoid arthritis: association with clinical features and treatment outcomes. Front Immunol 2018;9: 2351.

23. Moher D, Liberati A, Tetzlaff J, Altman DG; PRISMA Group. Preferred reporting items for systematic reviews and meta-analyses: the PRISMA statement. PLoS Med 2009;6: e1000097.

24. Hozo SP, Djulbegovic B, Hozo I. Estimating the mean and variance from the median, range, and the size of a sample. BMC Med Res Methodol 2005;5:13.

25. Ridout KK, Ridout SJ, Price LH, Sen S, Tyrka AR. Depression and telomere length: a meta-analysis. J Affect Disord 2016;191:237-47.

26. Cohen J. Statistical power analysis for the behavioral sciences. 2nd ed. Hillsdale (NJ), Lawrence Erlbaum, 1988.

27. Egger M, Smith GD, Phillips AN. Meta-analysis: principles and procedures. BMJ 1997;315:1533-7.

28. DerSimonian R, Laird N. Meta-analysis in clinical trials. Control Clin Trials 1986;7:177-88.

29. Higgins JP, Thompson SG. Quantifying heterogeneity in a meta-analysis. Stat Med 2002;21:1539-58.

30. Egger M, Davey Smith G, Schneider M, Minder C. Bias in meta-analysis detected by a simple, graphical test. BMJ 1997;315:629-34.

31. Bolon B. Cellular and molecular mechanisms of autoimmune disease. Toxicol Pathol 2012;40:216-29.

32. Cavalli G, Koenders M, Kalabokis V, Kim J, Tan AC, Garlanda C, et al. Treating experimental arthritis with the innate immune inhibitor interleukin-37 reduces joint and systemic inflammation. Rheumatology (Oxford) 2016;55: 2220-9. 